\title{
CHARACTERIZATION OF A PWT PHOTOINJECTOR AT HEAVY BEAM LOADING*
}

\author{
D. Yu, A.V. Smirnov, D. Newsham, Y. Luo, J. Zeng, DULY Research Inc. \\ Rancho Palos Verdes, CA 90275 \\ H. H. Braun, CERN, Geneva, Switzerland
}

\begin{abstract}
Performance of a single-section, S-band, Plane-WaveTransformer (PWT) photoelectron linac is characterized by a high (multi-ampere) average current required for advanced applications such as Compton x-ray sources and linear collider. Single bunch dynamics and multi-bunch beam loading effects are considered. Threshold current for regenerative $\mathrm{BBU}$ is calculated using a generalized Panofsky-Wenzel theorem, including such effects as the Lorentz' force component of the transverse wake field, inhomogeneous solenoidal focusing field, high relative energy gain and interaction with the waves of different polarizations. Stimulated regenerative BBU is considered for cumulative BBU.
\end{abstract}

\section{INTRODUCTION}

Most linac applications require high average bunch repetition rate superseding the operation rate of the modulator supplying power to the klystrons. In these cases the photoinjector must operate with multiple electron bunches in each rf pulse. For example, a Compton backscattering $\mathrm{x}$-ray source should have a minimum $1 \mathrm{kHz}$ rate [1]; next generation accelerators and colliders can have effective rates over $10 \mathrm{kHz}$ [2]. Along with significant charge per bunch $q_{b} \sim \mathrm{nC}$, it can result in severe problems caused by multi-bunch beam loading and BBU effects.

Feasibility of the S-band PWT photoinjector [3] for these modern applications is demonstrated in terms of single bunch characteristics and multi-bunch, high-current effects. The DULY S-band PWT was analyzed for application as an injector to both the Compton $\mathrm{x}$-ray source and linear collider. The Compton $\mathrm{x}$-ray source operates most efficiently [1] with a charge per bunch of approximately $0.5 \mathrm{nC}$. This charge is near the design specifications of the DULY S-band PWT, and previous simulations [3] indicate agreement with single bunch requirements of the $\mathrm{x}$-ray source.

\section{INJECTOR DESIGN}

We consider performance of the S-band PWT as a first stage of an injector to linear colliders. Analogous to the CTF3 design [2] we assumed $t_{b}=1.54 \mu \mathrm{s}$ beam pulse duration. Note, charge per bunch is $q_{b}=2.33 \mathrm{nC}$ significantly higher than that for the existing DULY design. We omit here the technical solutions and details of an rf system for coupling power into the resonator as well as laser facility providing emission of 3 ps bunches with $1.5 \mathrm{GHz}$ repetition frequency. Fig. 1 shows a preliminary design of the accelerator section proposed for this application. Solenoidal coils can provide maximum onaxis magnetic field up to $B_{z \max }=0.3 \mathrm{~T}$ (no field at the cathode). The $3 \mathrm{GHz} \pi$-mode PWT injector ( $30 \mathrm{~cm}$ long) consists of $5+2 / 2$ cells formed by disks suspended and cooled by 4 tubes in a large tank. To handle higher bunch charge we increase the aperture radius up to $a=1.214 \mathrm{~cm}$. The shunt impedance per unit length $r / Q_{o}=1833 \Omega / \mathrm{m}$, unloaded $Q$-factor $Q_{o}=25800$, and overvoltage ratio 1.72 are defined primarily from full-scale GdfidL [4] simulations. The calculated frequency separation of $230 \mathrm{MHz}$ between adjacent $\pi$ and $0.833 \pi$ modes is high due to large coupling between cells (in excess of 27\%) for this type of structure [5].

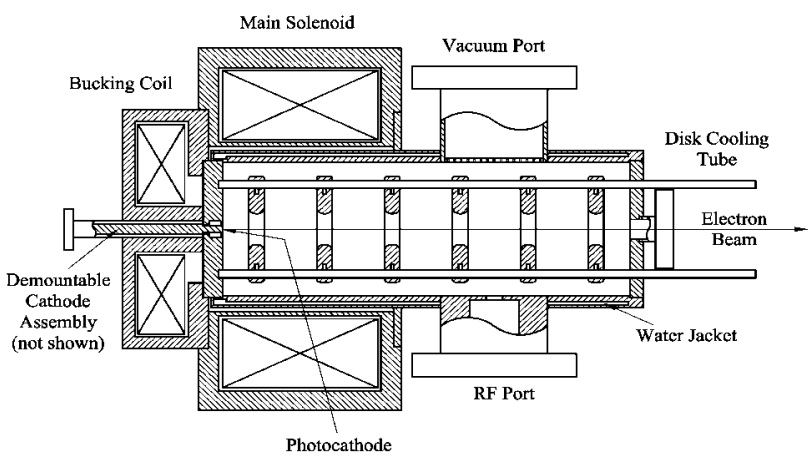

Figure 1: Schematic drawing of a high-current S-band PWT injector.

\section{MAIN PARAMETERS OF COMPENSATED BEAM LOADING}

Since the group velocity is zero, one can anticipate absence of multiple reflected waves during transient beam loading that occur in conventional structures with nonzero group velocity. Since the cells/half-cells are identical and the beam is well-bunched and relativistic, we can treat the structure as a single cavity loaded by a relativistic beam, omitting calculations for phase motion, bunch dimensions, short-range wakefields and diffused propagation of non-uniformity of the field induced. This non-flatness of the amplitude can result, e.g., from

* This work is supported by DOE SBIR No. DE-FG03-96ER82156. 
difference in beam loading in the first half-cell caused by lesser transit-time factor. The insignificant difference can be compensated by increasing the cell length. In a more general case (e.g., tapered structure and/or low rf power) we should take into account field amplitude and phase redistribution at diffused beam loading [6,7]. Under these conditions and for a given input power $P$ and beam current $I_{b}$ one can find analytically the optimal values of coupling coefficient $\beta_{c}$ and beam injection time $t_{o}$ providing constant energy gain (see Fig. 2):

$$
\begin{aligned}
& \beta_{c}=1+I_{b} R / U, U=\sqrt{\left(I_{b} R / 2\right)^{2}+P R}-I_{b} R / 2, R=r L, \\
& t_{o}=Q_{0} \ln \left(2 \beta_{c} /\left(\beta_{c}-1\right)\right) /\left(\pi f\left(\beta_{c}+1\right)\right) .
\end{aligned}
$$

Full compensation of beam loaded energy spread is one of the advantages of a $\pi$-mode PWT injector over a TW structure having inherent energy spread caused by transient beam loading [2]. Parameters found for this regime with compensated energy spread are given in Table 1 for two variants. We determined the deviation of each of the main parameters that results in an uncompensated energy spread $\Delta W=0.5 \mathrm{MeV}$ during $t_{b}$ (due to non-uniform beam loading): injection time jitter $\Delta t_{o}=0.03 \mu \mathrm{s}$, coupling coefficient inaccuracy of $\Delta \beta_{c}=0.065$ and beam current instability $\Delta I_{b}=0.25 \mathrm{~A}$. Note, the klystron power may exceed the calculated power $P$ because of the matching the klystron and accelerating resonator with special rf system.

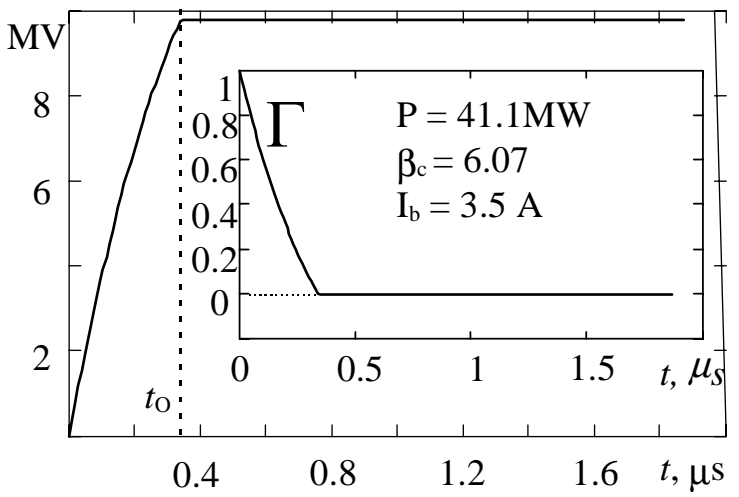

Figure 2: Effective accelerating voltage $U$ and absolute value of reflection coefficient (on the inset) versus time.

Table 1: PWT photoinjector parameters in multi-bunch compensated regime for beam current $I_{b}=3.5 \mathrm{~A}$.

\begin{tabular}{|l|c|c|}
\hline Input RF Power $P, \mathrm{MW}$ & 28 & 41.1 \\
\hline Peak on-axis field (loaded), MV/m & 50 & 70 \\
\hline RF pulse length $t_{r}, \mu \mathrm{s}$ & 1.79 & 1.88 \\
\hline Injection time $t_{o}, \mu \mathrm{s}$ & 0.25 & 0.34 \\
\hline Coupling coefficient $\beta_{c}$ & 8.1 & 6.07 \\
\hline Beam energy $W, \mathrm{MeV}$ & 6.9 & 9.8 \\
\hline \multicolumn{3}{|c|}{ PARMELA results, $q_{b}=2.33 \mathrm{nC} ;$ no detuning } \\
\hline Bunch length, mm & $<0.9$ & $<0.5$ \\
\hline Emittance (norm), mm.mrad & $<15$ & $<10$ \\
\hline Energy spread $\Delta W, \mathrm{MeV}$ & $<0.35$ & $<0.35$ \\
\hline Beam radius, $\mathrm{mm}$ & $<1.5$ & $<1.5$ \\
\hline
\end{tabular}

\begin{tabular}{|l|l|l|}
\hline Peak magnetic field $B_{\text {zmax }}, \mathrm{T}$ & 0.153 & 0.179 \\
\hline
\end{tabular}

Once the effective accelerating voltage is found and is constant during the pulse length, we can simulate single bunch dynamics that correspond to the multi-bunch steady-state mode of operation. The results of PARMELA [8] simulation are given in Table 1, Fig. 3.
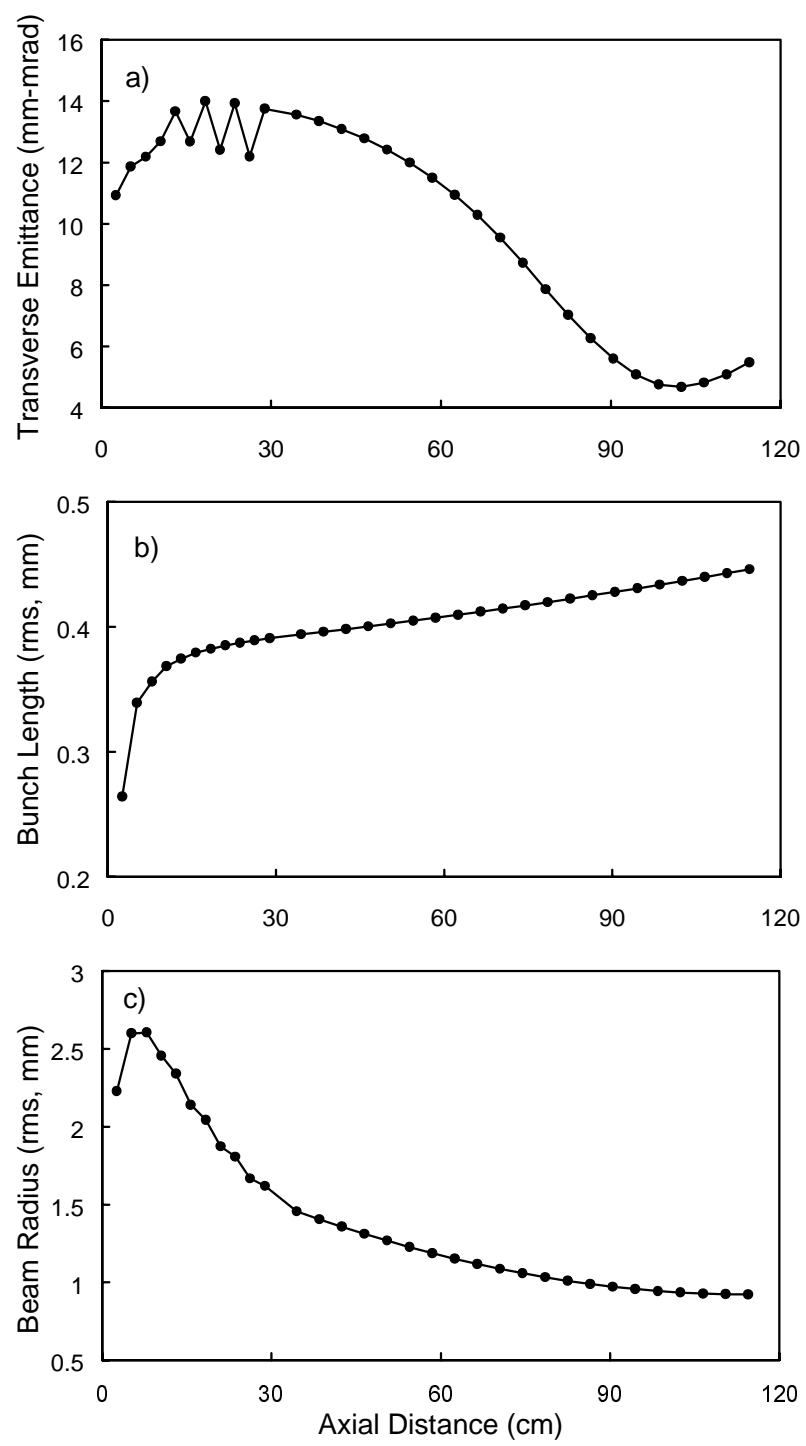

Figure 3: PARMELA plots for rms values of normalized emittance (a), bunch length (b); and beam radius ( c ).

\section{BEAM BREAK-UP}

In the injector design we deal with challenging requirements of high current, long pulse and low energy beams that can cause BBU effect. High acceleration rate and short length of the PWT injector are the key beneficial factors with respect to conventional TW designs. We analyze regenerative BBU effect in terms of threshold current with an extended theory developed for single section linac [9]. The main features included in the model are Lorentz' force component of the transverse wakefield, two-fold polarization of the dipole mode, real profiles of beam energy and longitudinal/transverse magnetic field. 
The model used here is based on Vainshtein's approach [10] of cavity excitation in terms of eigenmodes along with self-consistent transverse dynamics of the beam interacting with two dipole mode having the same frequency $f_{r}=c k_{r} / 2 \pi$ and different polarizations with $90^{\circ}$ angular shift. The generalization of Panofsky-Wenzel theorem was introduced [9] to include the effect of Lorentz' force component and hybrid coupling appearing when the phase slippage $\theta_{r}=k_{r} L\left(\beta_{r}^{-1}-\beta^{-1}\right)$ between the wave and beam is not zero. From GdfidL full-length and single-cell simulations we found the corresponding mode of the lowest dipole band with $f_{r}=3.78 \mathrm{GHz}$, minimal positive slippage $\theta_{r}=1.3$, unloaded Q-factor $Q_{r}=13515$, and transverse impedance per unit length $r_{\perp} / Q_{r}=178 \Omega / \mathrm{m}$ (see [11] for the $r_{\perp}$ definition). This model results in the following formula for the threshold current:

$I_{t h r}=\frac{2 m_{0} c^{2}}{\gamma_{r}^{2} e r_{\perp}} \cdot \frac{k_{r}^{-1}}{L^{2}} \cdot \frac{G\left(\Phi_{\gamma r}\right)\left(1+\beta_{c r}\right) \beta^{2} / \beta_{r}{ }^{2}}{\Psi_{r}\left(1-\beta \beta_{r}+\Xi_{r}\left(\beta-\beta_{r}\right)\right)}$,

where $\Psi_{r}$ is the bunch formfactor with respect to the dipole mode, $\beta=v / c, \beta_{r}=2 \pi f_{r} / c h_{r}, \gamma_{r}=1 / \sqrt{1-\beta_{r}{ }^{2}}$, $\beta_{c r}$ is the resonator coupling coefficient for the dipole mode, $\Xi_{r}$ is the hybrid coupling coefficients defined as: $\Xi_{r} / Z_{0}=-\left(\partial H_{z r}^{0} / \partial y\right) /\left(\partial E_{z r}^{0} / \partial x\right) \approx\left(\partial H_{z r}^{0} / \partial x\right) /\left(\partial E_{z r}^{0} / \partial y\right)$, $Z_{0}=\sqrt{\mu_{o} / \varepsilon_{o}}, G\left(\Phi_{\gamma r}\right)$ is the root of the equation: $G \operatorname{Re} \tilde{\Phi}_{\gamma_{r}}+\left|\operatorname{Im}\left(\sqrt{\left(G \operatorname{Im}_{j} \tilde{\Phi}_{\gamma r}\right)^{2}-((1-\alpha) / 2)^{2}}\right)\right|=(1+\alpha) / 2$, where $\alpha$ is the ratio between loaded Q-factors for the modes of two different polarizations,

$\widetilde{\Phi}_{\gamma r}\left(\theta_{r}\right)=\int_{0}^{1} \tilde{\varphi}_{\gamma}\left(\theta_{r}, \xi\right)\left(\exp \left(-i \theta_{r} \xi\right)\right) d \xi$, and $\tilde{\varphi}_{\gamma}(\xi)$ are scalar hypercomplex values defined by the equation:

$\left(\gamma \tilde{\varphi}_{\gamma}^{\prime}\right)^{\prime}=\exp \left(i \theta_{r} \xi\right)-j\left(\widetilde{\varphi}_{\gamma}^{\prime} \Omega+\tilde{\varphi}_{\gamma} \Omega^{\prime} / 2\right), \tilde{\varphi}_{\gamma}(0)=0=\tilde{\varphi}^{\prime}(0)$, where $i^{2}=-1, j^{2}=-1, i j \neq-1$, the prime means derivative $d / d \xi$, and $\Omega(\xi)=e B_{z}(L \xi) L / m_{o} c$. For conservative estimation of the threshold current we assumed $\beta_{c r}=0$, $\Xi_{r}=\Psi_{r}=\alpha=1$. It gives us the threshold current $I_{t h r}=4.52 \mathrm{~A}$. Note, in the presence of a solenoidal magnetic field the threshold current is finite at zero slippage and comparable with that at non-zero slippage.

The model built allows us to characterize the sensitivity of BBU to the input noise produced by the beam or input rf signal at the frequencies $f_{s}$ close to $f_{r}$. When the operating current is below threshold, the signal amplification gain can be estimated as follows: $K \approx\left|1+\Phi_{v r} I G /\left(1+i a_{r}-\Phi_{v r} I G\right)\right|$, where $a_{r}=2 Q_{r}\left(f_{r} / f_{s}-1\right)$, $\Phi_{v r}=\operatorname{Re}_{j} \tilde{\Phi}_{\gamma_{r}}+i\left(\operatorname{Im}_{j} \tilde{\Phi}_{\gamma r}\right)$ sign $\left(\operatorname{Im}_{j} \operatorname{Im}_{i} \tilde{\Phi}_{\gamma r}\right)$. Characteristic features of the gain curve depicted in Fig. 4 are confirmed experimentally [12] and are similar to an FEL linear gain curve. So, the stimulated BBU effect can amplify input signal even below the threshold. This is very important for cumulative BBU where even a very small relative gain in each section can cause emittance dilution or beam loss downstream the multi-section linac.

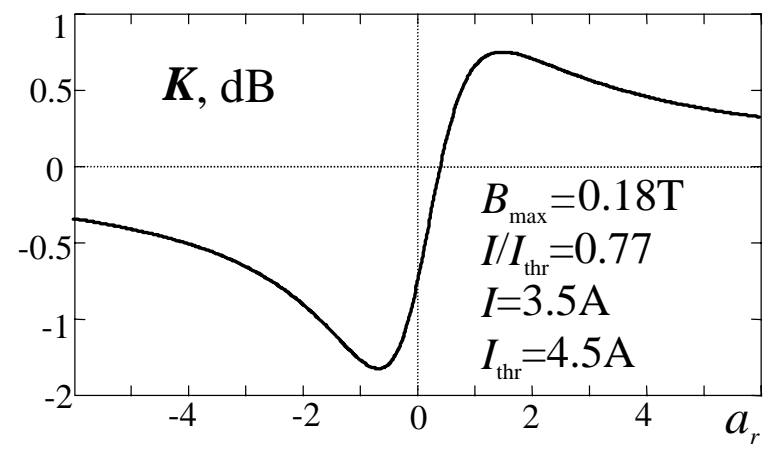

Figure 4: Amplification gain for the dipole mode versus normalized detuning $a_{r}=2 Q_{r}\left(f_{r} / f_{s}-1\right)$.

\section{REFERENCES}

[1] F.V. Hartemann et al., "Three-dimensional theory of emittance in Compton scattering and $\mathrm{x}$-ray protein crystallography," to be published in Phys. Rev. E. [2] L. Rinolf (Ed.) Proceedings of the $4^{\text {th }}$ CTF3 collaboration meeting, CLIC Note 433, May 2000. [3] D. Yu et al., Proc. of Particle Accelerator Conf., Vancouver, B.C. Canada, May 1997, p.2802; D. Yu et al., Proc. of Particle Accelerator Conf., New York, NY, March 1999, p.2203.

[4] W. Bruns, in Proc. of Particle Accelerator Conf. (PAC 97), Vancouver, B.C., Canada 12-16 May 1997, p. 2651.

[5] V.G. Andreev, Sov. Phys.-Technical Physics, v.13, N8 (1968) 1070.

[6] A.V. Smirnov, "Long Range Resonance Wake Fields at High Group Velocity and High Dispersion of Group Velocity," a talk given in the Accelerator Theory Club for ARDA group at SLAC. 02/09/01.

[7] P. B. Wilson, private communication.

[8] L. M. Young and J. H. Billen, PARMELA

Documentation, Report LA-UR-96-1835, revised December, 2000, Los Alamos National Laboratory. [9] A. V. Smirnov, "Effects of Beam Interaction with Fundamental and Asymmetric Modes in Tapered Linac Sections," Ph. D. Thesis, Moscow, Moscow Engineering Physics Institute, MEPhI (1985).

[10] L. A. Vainshtein, V.A. Solntsev, "Lectures on RF Electronics," Izdatelstvo “Sov. Radio,” Moscow (1973).

[11] P. Wilson, Preprint SLAC-PUB-2884 (1991).

[12] A. I. Zykov, G.D. Kramskoy, G.L. Fursov, in Pribory i Technika Experimenta, PTE, v. 5, N 28, (1971) p. 28 (In Russian). 